\title{
Improving Water Pressure Measurement Using Temperature-Compensated Wireless Passive SAW Bidirectional RDL Pressure Sensor
}

\author{
Zhaozhao Tang, Wenyan Wu, Jinliang Gao, Po Yang, Senior Member, IEEE, Ambreen Hussain, \\ Jingting Luo, Ran Tao, Chen Fu, and Tianli Li
}

\begin{abstract}
Currently pressure sensors utilized for water pressure measurement need batteries for direct power supply. However, batteries are lifespan-limited and not so reliable in the buried water pipe environment. The maintenance work due to battery failures leads to high cost to utility owners. Wireless passive surface acoustic wave (WP-SAW) sensors do not need direct power supply from batteries and can work in harsh environment. They are low-cost and compatible with microelectromechanical systems (MEMS) technologies. This study investigates a temperature-compensated WP-SAW bidirectional reflective delay line (RDL) pressure sensor and its feasibility in improving water pressure measurement. The linear temperaturecompensated pressure sensing functional model between the output phase shifts and the pressure change is established theoretically and verified by experiments. An experimental framework for testing the sensor node is built. The water pressure sensing adaptor is proposed. Experimental results: the experimental data show good linearities, which fits the established functional relationship; the numerical functional relationship has been derived and expressed; the sensor node has a good performance in the range of pressure difference from 0 to $0.5 \mathrm{MPa}$, which meets the normal 28-meter water pressure sensing requirements; the accuracy of this sensor is $7.22 \mathrm{kPa}$, which can be utilized for the water pressure sensing tasks in water distribution systems.
\end{abstract}

Index Terms - Surface acoustic wave (SAW), reflective delay line (RDL), sensor, water pressure, measurement
This work was supported in part by the EU FP7 - WatERP (318603), in part by the EU FP7 Marie Curie Actions - SmartWater (318985), in part by the EU Horizon 2020 Marie Skłodowska-Curie Actions - ITN - IOT4Win (765921), and in part by the Innovate UK (TS/V002953/1 and TS/W005913/1). (Corresponding author: Wenyan $\mathrm{Wu}$ )

Zhaozhao Tang is currently with the Key Laboratory of Optoelectronic Devices and Systems, College of Physics and Optoelectronic Engineering, Shenzhen University, Shenzhen, 518060, China, and he was previously with the School of Computing and Digital Technologies, Staffordshire University, Stoke on Trent, ST4 2DE, UK (e-mail: zhaozhao.tang@foxmail.com).

Wenyan $\mathrm{Wu}$ is currently with the School of Engineering and the Built Environment, Birmingham City University, Birmingham, B4 7XG, UK, and she was previously with the School of Computing and Digital Technologies, Staffordshire University, Stoke on Trent, ST4 2DE, UK (Corresponding author's e-mail: wenyan.wu@bcu.ac.uk).

\section{INTRODUCTION}

$\mathrm{T}$ HE water loss worldwide has been estimated to be as high as 126 billion cubic meters annually, which can cost 39 billion US dollars [1]. Drinking water loss caused by water pipe leakage in water distribution systems may reach up to $50 \%$ of the input in cases of insufficient maintenance [2]-[4]. Leakage in water distribution systems causes not only water loss, but also energy waste and contaminations to drinking water and environments which threatens human health [4]-[6]. Therefore, it becomes critical to efficiently detect and effectively reduce leakages. These tasks rely on proactive awareness of leaking points and prediction of potential water loss in good time.

Leakage detection has been investigated for many years. Modelling of water distribution systems and developing leakage detection algorithms are common methods utilized in the recent years. For example, Wang et al. [7] developed a pressure-dependent model for fast and efficiently localizing leakages in water distribution networks. Investigation of utilizing new artificial intelligence (AI) technologies for leakage detection started to step onto the arena nowadays. For instance, Harmouche et al. [8] utilized association rules mining techniques for long-term monitoring of water distribution networks. Kang et al. [9] ensembled convolutional neural network - support vector machine (CNN-SVM) and graphbased localization in water distribution systems for leakage detection. However, the aforementioned investigations encounter the bottleneck that only harnessing algorithms into processing water pressure data cannot make revolutions on the fundamental hardware systems to improve water pressure

Jinliang Gao is with the School of Environment, Harbin Institute of Technology, Harbin, 150090, China (e-mail: gil@hit.edu.cn).

Po Yang is with the Department of Computer Science, The University of Sheffield, Sheffield, S10 2TN, UK (e-mail: po.yang@sheffield.ac.uk).

Ambreen Hussain is currently with the School of Computing and Digital Technology, Birmingham City University, Birmingham, B4 7XG, UK, and she was previously with the School of Computing and Digital Technologies, Staffordshire University, Stoke on Trent, ST4 2DE, UK (e-mail: ambreen.hussain@bcu.ac.uk).

Jingting Luo, Ran Tao, and Chen Fu are with the Key Laboratory of Optoelectronic Devices and Systems, College of Physics and Optoelectronic Engineering, Shenzhen University, Shenzhen, 518060, China (e-mail: luojt@szu.edu.cn; ran.tao@szu.edu.cn; chenfu@szu.edu.cn).

Tianli Li is with the Shenzhen Key Laboratory of Electromagnetic Control, College of Mechatronics and Control Engineering, Shenzhen University, Shenzhen, 518060, China (email: 1t1988@gmail.com). 
measurement.

In order to improve water pressure measurement, novel pressure sensor systems are worthy of investigation. Currently pressure sensors utilized for water pressure measurement are so-called active sensors. They need batteries for direct power supply. Batteries are lifespan-limited and not so reliable in the buried water pipe environment. Thus, the maintenance work due to battery failures leads to high cost to utility owners. As for the problems of batteries and further improve reliability, wireless passive surface acoustic wave (WP-SAW) sensors are considered. They have numerous advantages over classic active sensors. For instance, they can be accessed wirelessly, do not need batteries for direct power supply, and enable remote measurement in harsh environment. Moreover, they have the rugged compact structure, outstanding stability, high sensitivity, rapid real time response, and extremely small size. They are low-cost and compatible to micro-electromechanical systems (MEMS) technologies [10][11]. They have been developed for a variety of industrial applications to measure physical parameters [12][13]. However, through reviewing large quantities of literatures, so far, only our preliminary studies reported the study of WP-SAW sensors for water pressure measurement.

In this paper, a temperature-compensated bidirectional WPSAW reflective delay line (RDL) pressure sensor and its feasibility in improving water pressure measurement is investigated. The sensor node based on a bidirectional RDL structure and connected to one antenna is introduced. The temperature-compensated pressure sensing functional relationship of the sensor node is established and verified by experiments. An experimental framework is built to test the sensor node and simulate the water pressure sensing adaptor, which can provide accurate and steady temperature and pressure in the test chamber. The experimental results are discussed, and show that the temperature-compensated bidirectional WP-SAW RDL pressure sensor is feasible on water pressure measurement with good accuracy.

In summary, this paper has the following main contributions.

1) SAW sensor is brought to water pressure measurement in water distribution systems. Compared to current water pressure sensor utilized in water distribution systems, this SAW sensor is a passive element and temperature-compensated, which are advantages over current water pressure sensors.

2) A linear temperature-compensated pressure sensing functional relationship between the output phase shifts and the pressure change for this bidirectional WP-SAW RDL pressure sensor node is established. It enables the elimination of ambient temperature interference to the sensor node.

3) Experimental evaluation and tests of the temperaturecompensated bidirectional WP-SAW RDL pressure sensor and experiments of water pipe simulator are implemented and discussed. The temperature-compensated bidirectional WPSAW RDL pressure sensor is feasible in improving water pressure measurement.

This paper is organized as follows. State of the art in WPSAW sensors is presented in Section II. The temperaturecompensated bidirectional WP-SAW RDL pressure sensor node is proposed in Section III. The linear temperaturecompensated pressure sensing functional relationship between the output phase shifts and the pressure change is established in Section IV. The experimental framework, water pressure sensing adaptor, and experimental steps are presented in Section V. Experimental results are discussed in Sections VI. Finally, the results are concluded in Section VII.

\section{StAte OF THE ART IN WP-SAW SENSORS}

The current state of the art in WP-SAW sensors includes two main types of architectures. They are SAW resonators and SAW RDLs. Both the two types utilize radio frequency (RF) signals for powering and interrogating sensor nodes. They both utilize interdigital transducer (IDT) on piezoelectric substates to convert interrogation RF signals into SAWs and convert reflected SAWs back to response RF signals. The differences are the reflectors and structures.

The SAW RDLs usually have several separate metal straps (RDLs) etched on a piezoelectric substrate for SAW reflections to produce different time delays on response RF signals. The measurands influence these time delays resulting in the phase shifts of response RF signals. Therefore, usually, the measurands are functions of phase shifts by utilizing WP-SAW RDL sensors. WP-SAW RDL sensors have been widely investigated for a number of applications. For instances, Xie et al. [14] introduced an embedded WP-SAW RDL pressure sensor with its design and package strategies for civil structure monitoring purpose. Devkota et al. [15] presented an $860 \mathrm{MHz}$ WP-SAW sensor with a metal-organic framework sensing layer for $\mathrm{CO}_{2}$ and $\mathrm{CH}_{4}$ measurement. Recently, new structures and interrogation schemes of WP-SAW RDL sensors have been investigated by researchers. For instance, Sveshnikov and Shitvov [16] presented a new architecture of WP-SAW RDL sensor tags with multi-transducers. Shi et al. [17] proposed a novel scheme called dual-frequency interrogation and hierarchical evaluation (DFI-HE) for resolving integer ambiguity of phase shifts and obtaining precise time delays between reflectors on WP-SAW RDL sensors.

The SAW resonators usually have Bragg reflectors which are formed by regular arranged metal straps etched on a piezoelectric substrate. Every strap only reflects a small quantity of SAWs. The superposition of the reflected SAWs allows frequency selection of different reflected SAW frequencies, which forms the resonant frequency of the SAW resonators. The measurands influence the resonant frequency on the response RF signals. Therefore, usually, the measurands are functions of resonant frequencies by utilizing WP-SAW resonator sensors. WP-SAW resonators have been developed for industrial applications, e.g., wind turbines [18], marine applications [19], automotive applications [20], etc. They have been utilized for monitoring temperature, strain, torque, pressure [21], etc. Researchers have been working for enhanced 


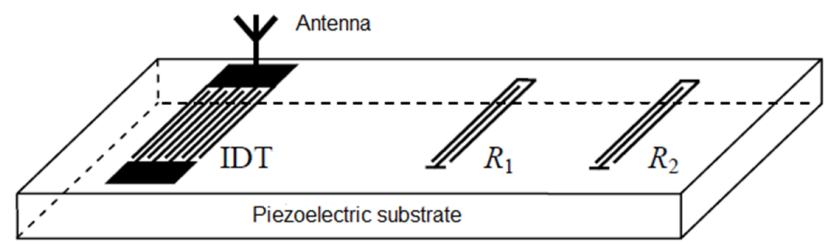

Fig. 1. The structure of a typical RDL type WP-SAW sensor node.

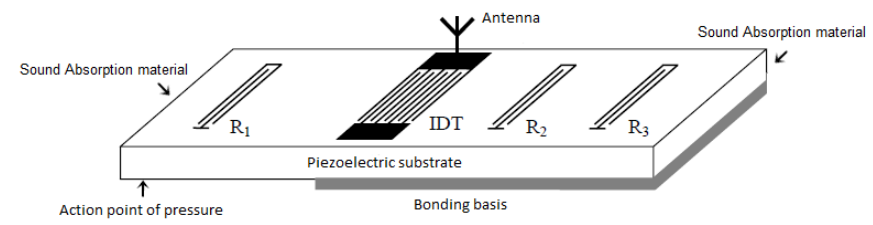

a)

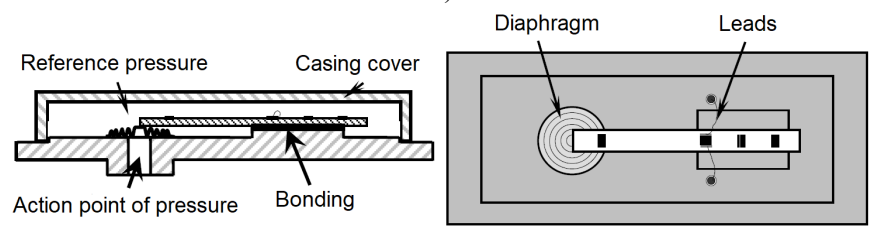

b)

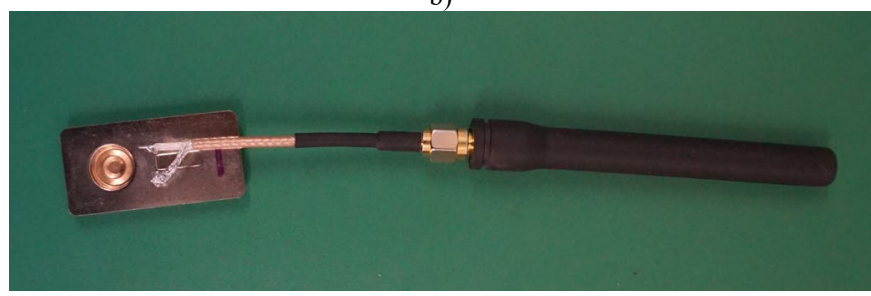

c)

Fig. 2. The temperature-compensated bidirectional WP-SAW RDL pressure sensor node: a) structure; b) casing; c) photo.

accuracy and reliability of WP-SAW sensors. For example, in order to detect sensors accurately and reliably, Yang et al. [22] investigated an adaptive environment-aware threshold determination algorithm for WP-SAW resonator sensors. Khoshakhlagh et al. [23] proposed a pigeonhole principle-based method for estimating the resonant frequency of WP-SAW resonator sensors. Lurz et al. [24] presented a new interrogation method for precise and fast frequency determination of WPSAW resonator sensors based on instantaneous frequency measurement by a low-cost six-port interferometer.

\section{ThE BIDIRECTIONAL WP-SAW RDL PRESSURE SENSOR NODE}

A conventional WP-SAW RDL sensor node consists of one IDT and several RDLs located at one side of the IDT. They are paralleled to the IDT bars and vertical to the direction of SAW propagation, as shown in Fig. 1.

The bidirectional WP-SAW RDL sensor node is shown in Fig. 2. The core elements of this sensor node are a piezoelectric substrate (a $0.5 \mathrm{~mm}$ thick $\mathrm{Y}-\mathrm{Z}$ cut $\mathrm{LiNbO}_{3}$ piezoelectric crystal), an IDT (Al) and three RDLs $\left(\mathrm{R}_{1}, \mathrm{R}_{2}\right.$ and $\left.\mathrm{R}_{3}\right)(\mathrm{Al})$ etched (conventional photolithographic lift-off process) on the surface of the substrate. The IDT is located in the center, and RDLs are located at both sides of the IDT. RDLs are paralleled to the centered IDT bars and vertical to two opposite directions of SAW propagation. This bidirectional RDL structure enables
TABLE I

PARAMETERS OF THE TEMPERATURE-COMPENSATED BIDIRECTIONAL WPSAW RDL PRESSURE SENSOR NODE

\begin{tabular}{cc}
\hline \hline Component name and unit & Parameters \\
\hline Center frequency $(\mathrm{MHz})$ & 433 \\
SAW wavelength $\lambda(\mu \mathrm{m})$ & 8 \\
Bar width $(\mu \mathrm{m})$ & 2 \\
Bar interval $(\mu \mathrm{m})$ & 2 \\
Bar length $(\mu \mathrm{m})$ & 440 \\
IDT width $(\mu \mathrm{m})$ & 400 \\
Thickness of metal Al $(\mu \mathrm{m})$ & 0.2 \\
Distance between IDT and $\mathrm{R}_{1}(\mu \mathrm{m})$ & 7000 \\
Distance between IDT and $\mathrm{R}_{2}(\mu \mathrm{m})$ & 2400 \\
Distance between IDT and $\mathrm{R}_{3}(\mu \mathrm{m})$ & 4800 \\
\hline \hline
\end{tabular}

full use of SAW energies at both opposite directions of SAW propagation.

An antenna is connected to the IDT for receiving and transmitting RF signals. After receiving RF signals (interrogation signals) from the reader (RF signal interrogating and receiving unit) by the antenna, the WP-SAW RDL sensor node is activated and the received RF signals are processed in two steps. Firstly, the IDT converts the received RF signals into SAWs which propagates on the surface of the piezoelectric crystal towards the RDLs at both sides of the IDT. Secondly, parts of the incoming SAWs are reflected by the specific patterned RDLs. The parts of reflected SAWs propagate to the IDT and are converted back into RF signals (response signals). Response signals are transmitted back by the antenna to the reader. The ambient interferences can change the propagation of the SAW, which results in time delay changes and further phase shifts of the response signals.

In order to sense pressure change, the measurand pressure must be able to create a pressure difference and make a deformation on the piezoelectric substrate. Before the measurand pressure acts on the piezoelectric substrate, the piezoelectric substrate must be kept at a constant pressure. Therefore, the piezoelectric substrate is strictly sealed in a metal cavity, and the right half of the lower surface is bonded and fixed to the inner housing. Thanks to this package strategy, the right half of the substrate is only sensitive to ambient temperature changes and free from pressure changes, and the left half of the substrate forming a cantilever is sensitive to both ambient temperature and pressure changes. The sensor node is shown in Fig. 2.

Based on the above design strategy, $\mathrm{R}_{2}$ is designed as a reference $\mathrm{RDL}, \mathrm{R}_{1}$ and $\mathrm{R}_{3}$ are designed for pressure sensing and temperature compensation purposes respectively. Pressure changes cannot be extracted by only processing the response signals related to $R_{1}$ and $R_{2}$, because the interference of temperature change is also effective on the cantilever. Therefore, a temperature-compensated pressure sensing functional relationship by processing all response signals related to $R_{1}, R_{2}$ and $R_{3}$ is established in the next section.

The SAWs reflected by substrate edges can overlap with the SAWs reflected by RDLs. The overlaps of SAW cause difficulties on subsequent signal processing and resolving sensing information in time domain. Therefore, in order to eliminate the interferences of the SAW reflected by substrate 


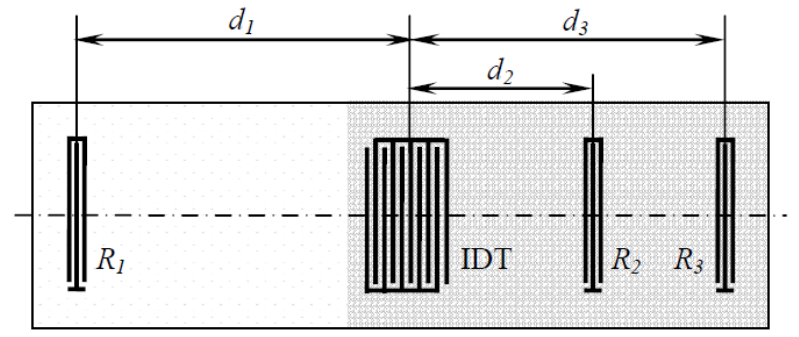

a) Top view

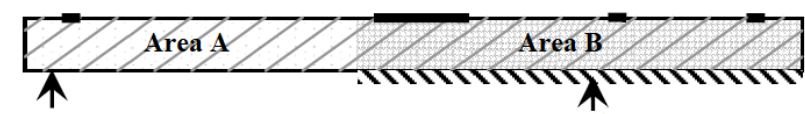

Action point of pressure

b) Side view

Bonding basis

Fig. 3. The plane figure of the temperature-compensated bidirectional WPSAW RDL pressure sensor node (the plane figure of Fig. 2): a) top view; b) side view.

edges, sound absorption materials are placed on both edges of the substrate to absorb excessive SAW energy. To reduce response signals overlapping with the noise caused by substrate edge reflections in time domain, the substrate edges should be far enough from the IDT, though edge reflection energies are mostly absorbed by sound absorption materials.

For the convenience of sensing information extraction, the effective response signals should be single-time-reflected SAW by the three RDLs. The intervals in time domain of interrogation signals should be long enough to make multireflected SAW attenuate thoroughly and separate effective single-time-reflected response signals for effective sensing information extraction.

After all factors are considered, the dimensions of all components of the sensor node are designed, which are shown in Table I.

\section{TEMPERATURE-COMPENSATED FUNCTIONAL RELATIONSHIP ESTABLISHMENT BETWEEN THE OUTPUT PHASE SHIFTS AND THE PRESSURE CHANGE}

Based on the working mechanism of the WP-SAW RDL sensor, phase differences between interrogation and response signals are influenced by four effects: measurands, initial setting state, transmission delay in the reader, and the distance between the reader and the sensor node antennas. In a practical measurement, the last effect cannot be accurately measured. Although the second and third effects can be measured, due to the sensor node structure, the last three effects are embodied synergistically, resulting in the incapability of measurement separately. A model must be built which can eliminate the last three effects, so that the time delay directly related to measurands can be accurately known.

Fig. 3 is the plane figure of the temperature-compensated bidirectional WP-SAW RDL pressure sensor, which shows some key parameters of the sensor node. Following is the stepby-step establishment of temperature-compensated functional relationship between the output phase shifts and the pressure change, which was firstly presented by us in [25] and improved in this paper.
The initial interrogation signal transmitted from the reader antenna $S(t)$ is given by equation (1).

$S(t)=A \cos \left(\omega_{0} t+\theta_{0}\right)=A \cos [\theta(t)]$

where $\omega_{0}$ is the initial angular velocity; $t$ is the time; $\theta_{0}$ is the initial phase of the interrogation signal; $A$ is the initial amplitude of the interrogation signal.

The response signal reflected by $R_{i}(i=1,2,3)$ is given by equation (2).

$S_{i}(t)=A_{i} \cos \left[\omega_{0}\left(t-t_{i}\right)+\theta_{0}\right]=A_{i} \cos \left[\theta_{i}(t)\right]$

where $t_{i}$ is the time delay of the response signal reflected by $\mathrm{R}_{\mathrm{i}}$ ( $\left.\mathrm{i}=1,2,3\right)$ against the interrogation signal; $A_{i}$ is the amplitude of the response signal reflected by $\mathrm{R}_{\mathrm{i}}(\mathrm{i}=1,2,3)$, which is a smaller value than $A$ due to attenuation of SAW propagation.

The time delay $t_{i}$ consists of two parts shown in equation (3), where $\tau_{i}$ is the time delay caused by the SAW propagation from the IDT to the $\mathrm{R}_{\mathrm{i}}(\mathrm{i}=1,2,3)$ and the back way to the IDT, and $\tau_{e}$ is the time delay caused by initial setting state, transmission delay in the reader, and RF signals propagating between the reader and the sensor node. $\tau_{e}$ is identical for all response signals. $\tau_{i}$ can be derived by $d_{i}$, the distance from the IDT to the $\mathrm{R}_{\mathrm{i}}(\mathrm{i}=1,2,3)$ and $v$, the velocity of the $\mathrm{SAW}$ propagation on the substrate.

$t_{i}=\tau_{i}+\tau_{e}=\frac{2 d_{i}}{v}+\tau_{e}$

From the sensor node structure, $\mathrm{R}_{2}$ and $\mathrm{R}_{3}$ are designed for reference and temperature compensation respectively. $\varphi_{T}$ is defined as the phase difference which indicates the temperature impact of the ambient environment, which is derived in equation (4). $\tau_{e}$ is taken out by the subtraction.

$$
\begin{aligned}
\varphi_{T}=\varphi_{3}-\varphi_{2}= & \omega_{0}\left(t_{3}-t_{2}\right)=\omega_{0}\left(\tau_{3}-\tau_{2}\right) \\
& =\omega_{0} \frac{2\left(d_{3}-d_{2}\right)}{v}=\omega_{0} \tau_{T}
\end{aligned}
$$

where $\tau_{T}$ is the round-trip time delay of the SAW propagation from $R_{2}$ to $R_{3}$ and reflected back from $R_{3}$ to $R_{2}$.

The relationship between $\tau_{T}$ and the temperature $T$ is shown is equation (5).

$\tau_{T}=\frac{2\left(d_{3}-d_{2}\right)}{v}=\frac{2 d_{T}}{v}=\tau_{T_{0}}\left[1+\alpha\left(T-T_{0}\right)\right]$

where $d_{T}$ is the distance between $\mathrm{R}_{2}$ and $\mathrm{R}_{3}$ on the surface of the sensor substrate; $\tau_{T_{0}}$ is the round-trip time delay caused by the SAW propagation from $R_{2}$ to $R_{3}$, and the back way from $R_{3}$ to $\mathrm{R}_{2}$ at the reference temperature $T_{0} ; \alpha$ is the temperature coefficient of the substrate material.

$\tau_{T_{0}}$ can be derived by equation (6) in which $d_{T_{0}}$ is the distance between $R_{2}$ and $R_{3}$ on the surface of the substrate at the reference temperature $T_{0}$. 
$\tau_{T_{0}}=\frac{2 d_{T_{0}}}{v}$

According to the propagation theory of SAW, the initial angular velocity $\omega_{0}$ is derived in equation (7), where $f_{0}$ is the initial frequency corresponding to $\omega_{0}$.

$\omega_{0}=2 \pi f_{0}$

Equation (8) shows the relationship between the SAW propagation velocity $v$ and $f_{0}$, in which $\lambda$ is the wavelength of the SAW propagating on the surface of the substrate.

$v=f_{0} \lambda$

$\varphi_{T_{0}}$ is defined as the phase difference directly indicating the temperature impact of the ambient environment at the reference temperature $T_{0}$. Equation (9) shows the relationship between $\varphi_{T_{0}}$ and $\tau_{T_{0}}$ in a similar way as equation (4).

$\varphi_{T_{0}}=\omega_{0} \tau_{T_{0}}$

Summarizing equations (4)-(5)(6)(7)(8)(9), the temperature change $\Delta T$ can be derived using the phase shift $\Delta \varphi_{T}$ which indicates the temperature impact of the ambient environment, shown in equation (10).

$\Delta T=T-T_{0}=\frac{\lambda}{4 \pi \alpha d_{T_{0}}}\left(\varphi_{T}-\varphi_{T_{0}}\right)=\frac{\lambda}{4 \pi \alpha d_{T_{0}}} \Delta \varphi_{T}$

The time delay $t_{1}$ is affected by both temperature and pressure changes. In order to extract the independent effect of pressure change from the response signal reflected by $R_{1}$, the interference effect caused by temperature change must be eliminated.

Similar to equation (4), $\varphi_{P T}$ is the phase difference between the response signals reflected by $R_{1}$ and $R_{2}$, which can be derived in equation (11).

$$
\begin{aligned}
\varphi_{P T}=\varphi_{1}-\varphi_{2}= & \omega_{0}\left(t_{1}-t_{2}\right)=\omega_{0}\left(\tau_{1}-\tau_{2}\right) \\
& =\omega_{0} \frac{2\left(d_{1}-d_{2}\right)}{v}=\omega_{0} \tau_{P T}
\end{aligned}
$$

where $\tau_{P T}$ is the SAW propagation time difference between the round trip from the IDT to $\mathrm{R}_{1}$ and reflected back to the IDT and the round trip from the IDT to $\mathrm{R}_{2}$ and reflected back to the IDT.

$\mu_{T}$ is defined as the average strain caused by temperature change, and $\mu_{P}$ is defined as the average strain caused by pressure change (see equations (12) and (13)).

$\mu_{T}=\alpha\left(T-T_{0}\right)=\alpha \Delta T$

$\mu_{P}=\beta\left(P-P_{0}\right)=\beta \Delta P$ where $\beta$ is the pressure coefficient of the substrate material; $P$ is the measurand pressure; $P_{0}$ is the reference pressure; $\Delta P$ is the pressure change.

$\tau_{P T}$ can be derived by equation (14).

$\tau_{P T}=\frac{2\left(d_{1}-d_{2}\right)}{v}=\frac{2 d_{P T}}{v}=\tau_{P_{0} T_{0}}\left(1+\mu_{P}+\mu_{T}\right)$

where $d_{P T}$ is the distance difference of $d_{1}$ and $d_{2} ; \tau_{P_{0} T_{0}}$ is the time difference at the reference temperature $T_{0}$ and reference pressure $P_{0}$, which can be derived by equation (15).

$\tau_{P_{0} T_{0}}=\frac{2 d_{P_{0} T_{0}}}{v}$

where $d_{P_{0} T_{0}}$ is the distance difference of $d_{1}$ and $d_{2}$ at the reference temperature $T_{0}$ and reference pressure $P_{0}$.

Summarizing equations (11)-(12)(13)(14)(15), $\varphi_{P T}$ can be derived by equation (16), and $\varphi_{P_{0} T_{0}}$ can be derived by equation (17).

$$
\begin{aligned}
\varphi_{P T}=\omega_{0} \tau_{P T}= & \omega_{0} \frac{2 d_{P_{0} T_{0}}}{v}\left(1+\mu_{P}+\mu_{T}\right) \\
& =\omega_{0} \frac{2 d_{P_{0} T_{0}}}{v}(1+\beta \Delta P+\alpha \Delta T) \\
\varphi_{P_{0} T_{0}}=\omega_{0} \tau_{P_{0} T_{0}} & =\omega_{0} \frac{2 d_{P_{0} T_{0}}}{v}
\end{aligned}
$$

where $\varphi_{P_{0} T_{0}}$ is the phase difference between the response signals reflected by $\mathrm{R}_{1}$ and $\mathrm{R}_{2}$ at the reference temperature $T_{0}$ and reference pressure $P_{0}$.

Therefore, $\Delta \varphi_{P T}$, the subtraction of $\varphi_{P T}$ and $\varphi_{P_{0} T_{0}}$ can be derived by equation (18).

$\Delta \varphi_{P T}=\varphi_{P T}-\varphi_{P_{0} T_{0}}=\omega_{0} \frac{2 d_{P_{0} T_{0}}}{v}(\beta \Delta P+\alpha \Delta T)$

Summarizing equations (7)-(8)(18), the pressure change can be derived as equation (19).

$\Delta P=P-P_{0}=\frac{\lambda}{4 \pi \beta d_{P_{0} T_{0}}} \Delta \varphi_{P T}-\frac{\alpha}{\beta} \Delta T$

Summarizing equations (10) and (19), the pressure change can be expressed by output phase shifts, as equation (20).

$\Delta P=\frac{\lambda}{4 \pi \beta}\left(\frac{\Delta \varphi_{P T}}{d_{P_{0} T_{0}}}-\frac{\Delta \varphi_{T}}{d_{T_{0}}}\right)=M \Delta \varphi_{P T}-N \Delta \varphi_{T}$

where $M$ and $N$ are constants.

In summary, the temperature-compensated functional relationship between the output phase shifts and the pressure change is established as equation (20), which shows a linear relationship. 


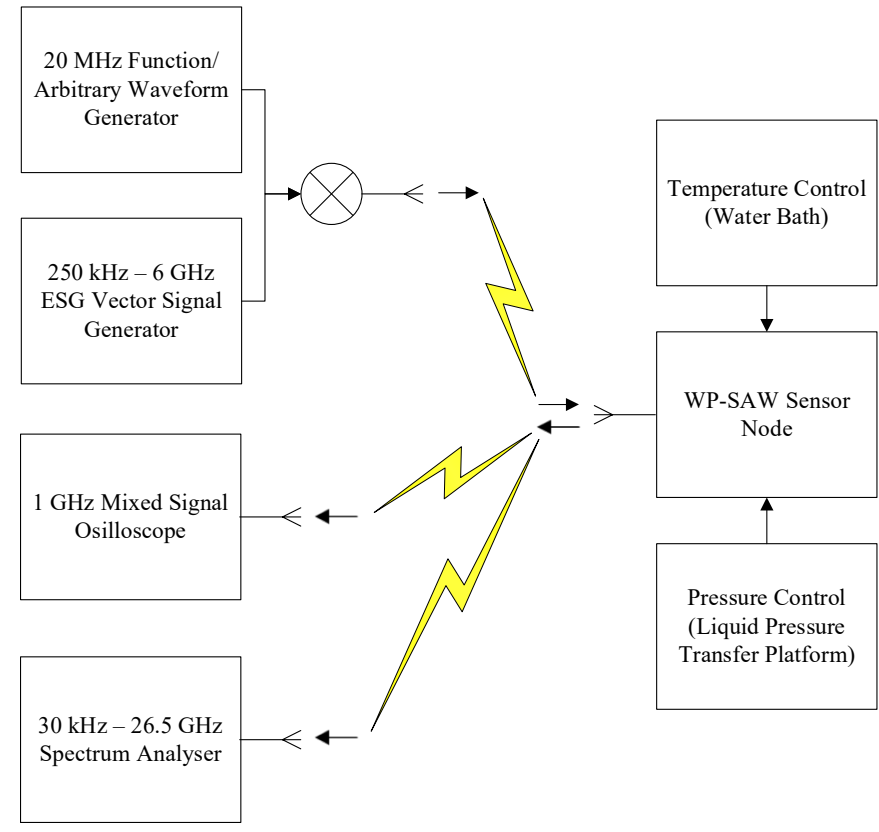

Fig. 4. The experimental framework and the RF signal flow for the performance evaluation of the temperature-compensated bidirectional WPSAW RDL pressure sensor node.

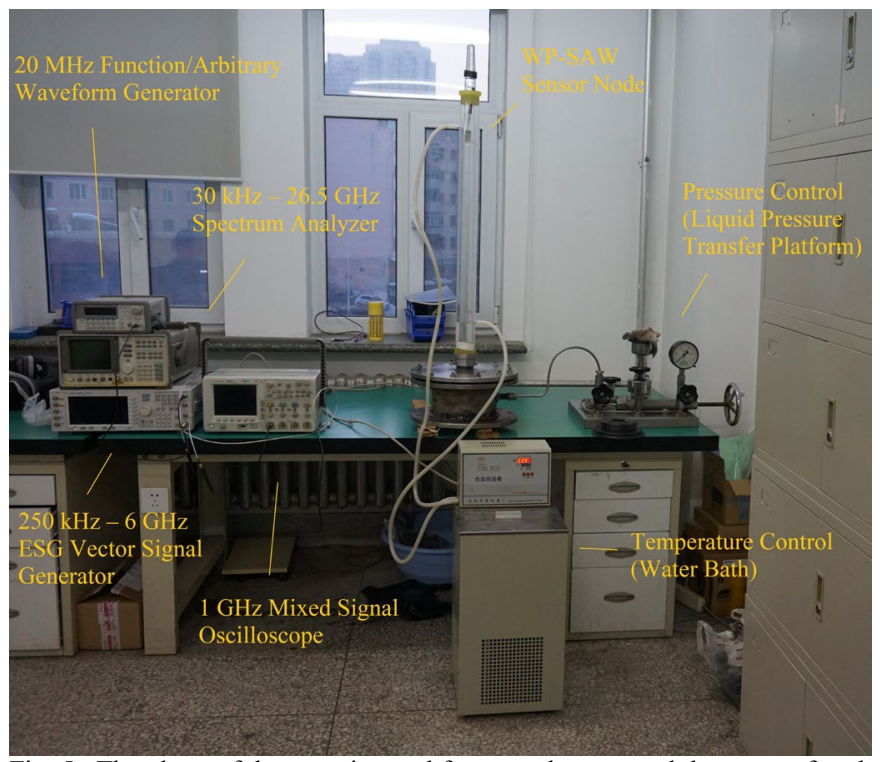

Fig. 5. The photo of the experimental framework setup and the name of each component.

\section{EXPERIMENTAL FRAMEWORK AND WATER PRESSURE SENSING ADAPTOR}

\section{A. Experimental Framework Setup}

Fig. 4 shows the experimental framework setup and RF signal flow for evaluating the performance of the WP-SAW sensor node. This structure was first presented by us in [26]. Fig. 5 is the photo of the experimental framework setup. It was previously presented by us in [26]-[28]. The instruments include a $20 \mathrm{MHz}$ Function/Arbitrary Waveform Generator (Agilent 33220A Function/Arbitrary Waveform Generator), a $250 \mathrm{kHz}-6 \mathrm{GHz}$ ESG Vector Signal Generator (Agilent E4438C ESG Vector Signal Generator), a $1 \mathrm{GHz}$ Mixed Signal
Oscilloscope (Agilent MSO 6104A Mixed Signal Oscilloscope) and a $30 \mathrm{kHz}-26.5 \mathrm{GHz}$ Spectrum Analyzer (Agilent E4440A PSA Series Spectrum Analyzer). The Function/Arbitrary Waveform Generator (Agilent 33220A) generates the pulse signal is multiplied by the standard $433 \mathrm{MHz}$ sine wave generated by the ESG Vector Signal Generator (Agilent E4438C). The multiplication result of the two signals is transmitted out as the interrogation signal to the WP-SAW sensor node. The sensor node receives interrogation signal from the signal generators and then reflect it to form response signals with sensing information to the spectrum analyzer and the oscilloscope. The signal generators, the spectrum analyzer and the oscilloscope work together to form the function of the reader for the WP-SAW sensor node.

The functions of each experimental instrument are as follows. The interrogation signal is generated by the multiplication of two signals (pulse and sine wave) generated by two signal generators. It activates the sensor node and is reflected by the three RDLs to form response signals. The oscilloscope and the spectrum analyzer receive both response and interrogation signals wirelessly and processes them in time and frequency domains respectively. The experimental framework includes a two-layer glass water pipe, a water bath temperature controller and a liquid pressure transfer platform. The temperature of the strictly sealed inner-layer glass pipe is controlled by the cycled water in the outer-layer glass pipe with constant temperature from the water bath container, and the pressure of the inner-layer glass pipe can be adjusted by the liquid pressure transfer platform. Based on Boyle's Law, the pressure applied in the strictly sealed inner-layer glass pipe via the liquid pressure transfer platform is equal to the air pressure sensed by the sensor node. The actual temperature and pressure values can be read from the water bath temperature controller and the liquid pressure transfer platform respectively, while the experimental values can be obtained through processing the interrogation and response signals.

During measurement, time delays of response signals are extremely small. For the instance of temperature compensation, $0.1{ }^{\circ} \mathrm{C}$ variation of temperature only causes $0.01 \mathrm{~ns}$ variation of time delay. If direct measurement were applied, the accuracy of

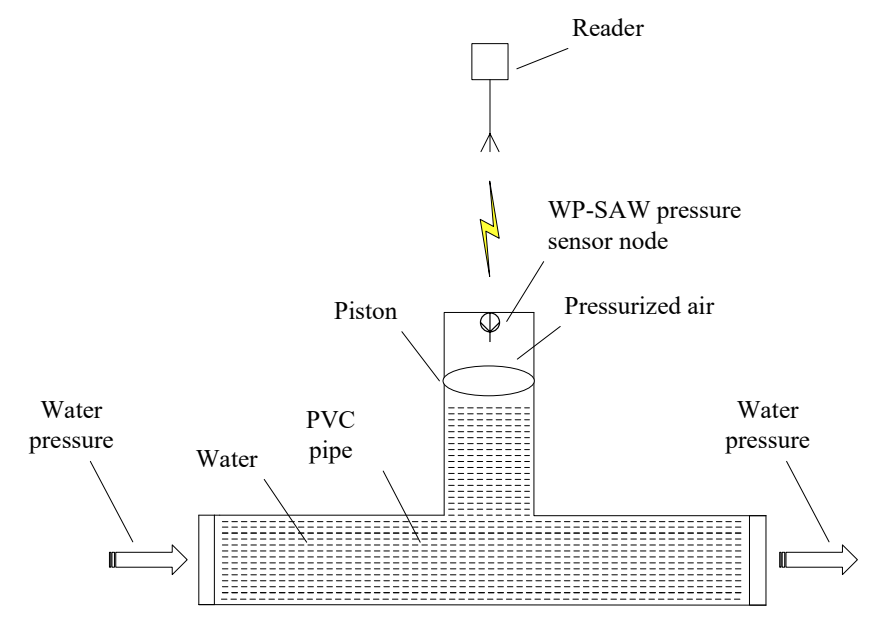

Fig. 6. The proposed water pressure sensing adaptor for WP-SAW sensor system. 

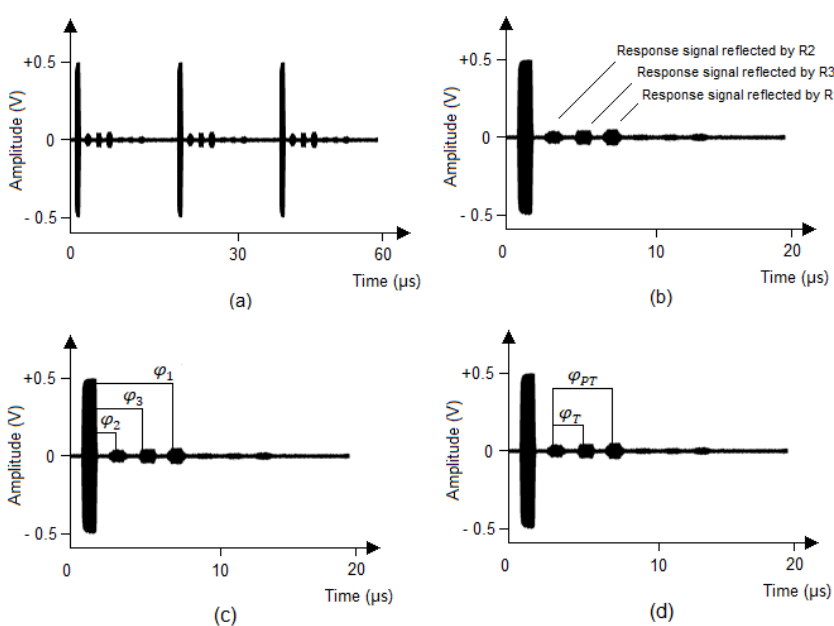

Fig. 7. The interrogation signal and three response signals reflected by three RDLs in time domain: (a) three sampling periods; (b) one sampling period; (c) phase differences between the three response signals to the interrogation signal; (d) temperature and pressure directly related phase differences.

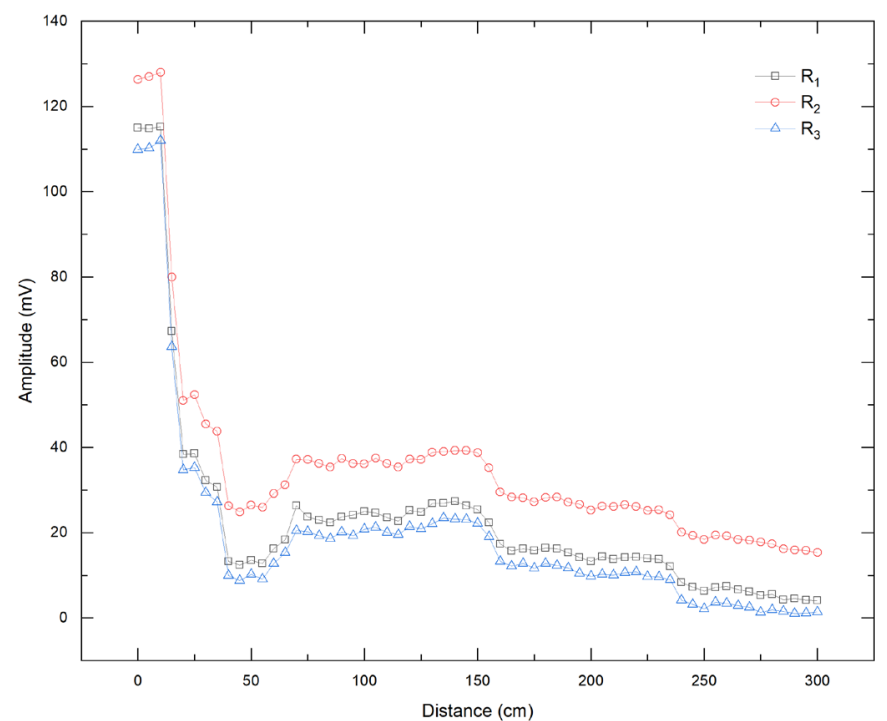

Fig. 8. The influence of interrogation distance on response RF signal amplitudes.

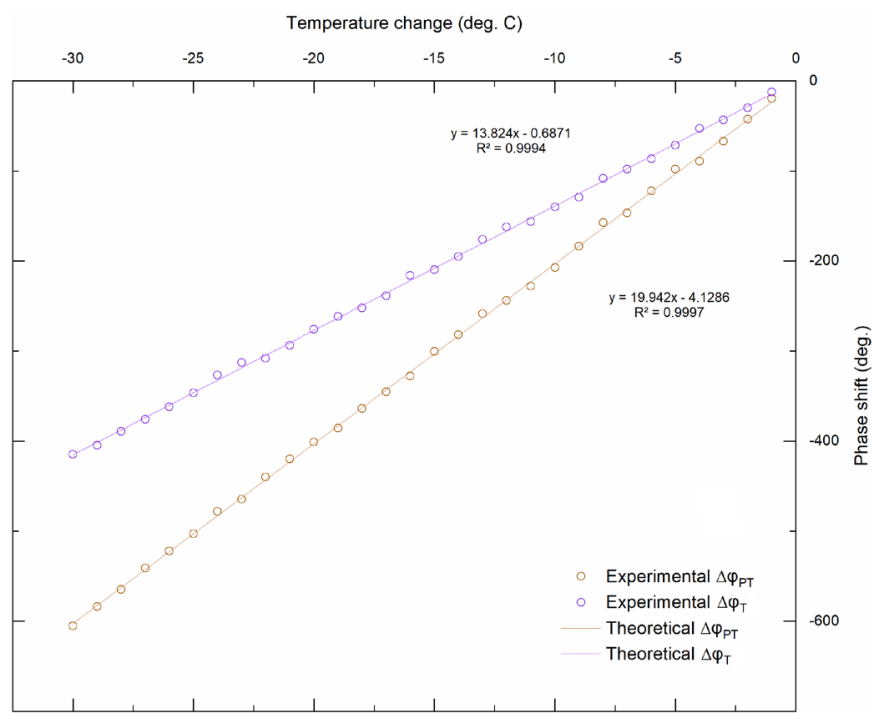

Fig. 9. Measurement data of output phase shifts $\left(\Delta \varphi_{T}, \Delta \varphi_{P T}\right)$ depending on the temperature change $\Delta T$ at constant pressure $1 \mathrm{~atm}$ (Reference temperature: $\left.55^{\circ} \mathrm{C}\right)$.

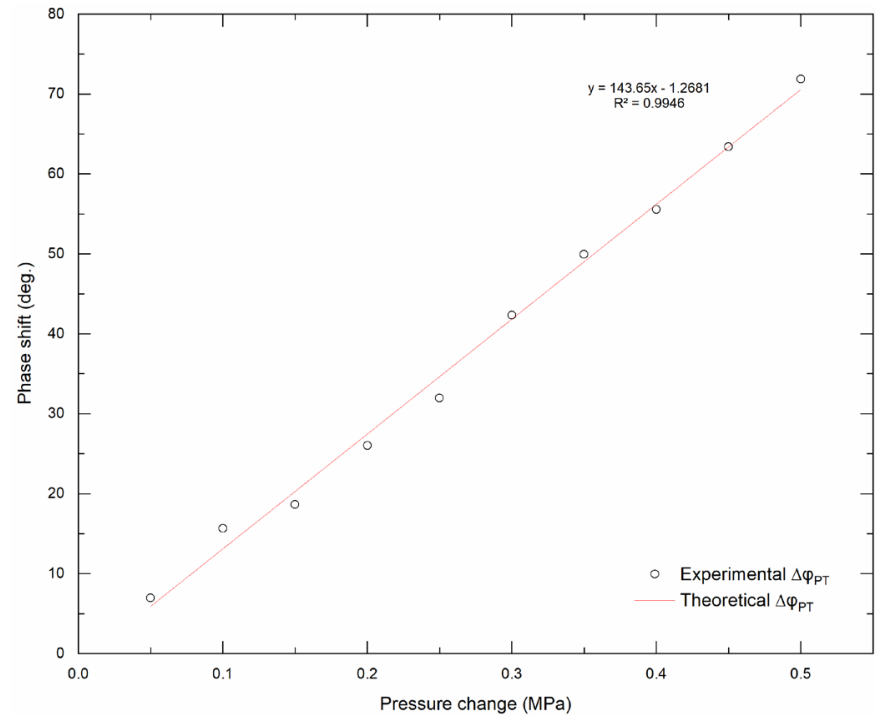

Fig. 10. Measurement data of output phase shift $\Delta \varphi_{P T}$ depending on the pressure change $\Delta P$ at constant temperature $25{ }^{\circ} \mathrm{C}$ (Reference pressure: 1 atm).

measuring instruments would have to reach picosecond level. This is difficult to be achieved by current hardware. However, phase shifts can be measured instead of direct measuring time delays because the time delay of a signal at a certain frequency is a function of the phase difference. One period time delay corresponds to $360^{\circ}$ phase difference. Generally, the frequency of an RF signal varies from several tens' $\mathrm{Hz}$ to several $\mathrm{GHz}$. A period of an RF signal is usually at nanoscale. It is easier to reach picosecond level by measuring phase shifts. To measure phase shifts, high frequency RF signals are processed through down conversion, low-pass filtering and quadrature sampling. Through these processes, phase shifts can be obtained from measured RF interrogation and response signals.

\section{B. Water Pressure Sensing Adaptor}

Showed in Fig. 6, inspired from the experimental framework, the water pressure sensing adaptor is proposed, which is improved from our previous one in [29]. The WP-SAW sensor node was attached to the top of the inner chamber of the adaptor. It can directly sense the pressurized air pressure above the piston. Similar to the experimental framework, according to the Boyle's Law, the pressurized air pressure above the piston is equal to the water pressure below the piston. Therefore, water pressure can be measured through pressurized air pressure in the sensor node chamber without contacting the water.

Due to the electromagnetic shielding effect of metals, this adaptor is only designed for non-metal material pipes, e.g., polyvinyl chloride (PVC). The RF interrogation signal emitted from the reader activates the WP-SAW pressure sensor node in the adaptor and the RF response signals with water pressure sensing information transmit back to the reader for processing. This sensor node is battery-free benefitting from its passive feature.

\section{EXPERIMENTAL RESULTS AND DISCUSSIONS}

The sensor node is initially characterized by the network analyzer (Keysight E5071C 100kHz - 4.5 GHz ENA Series 


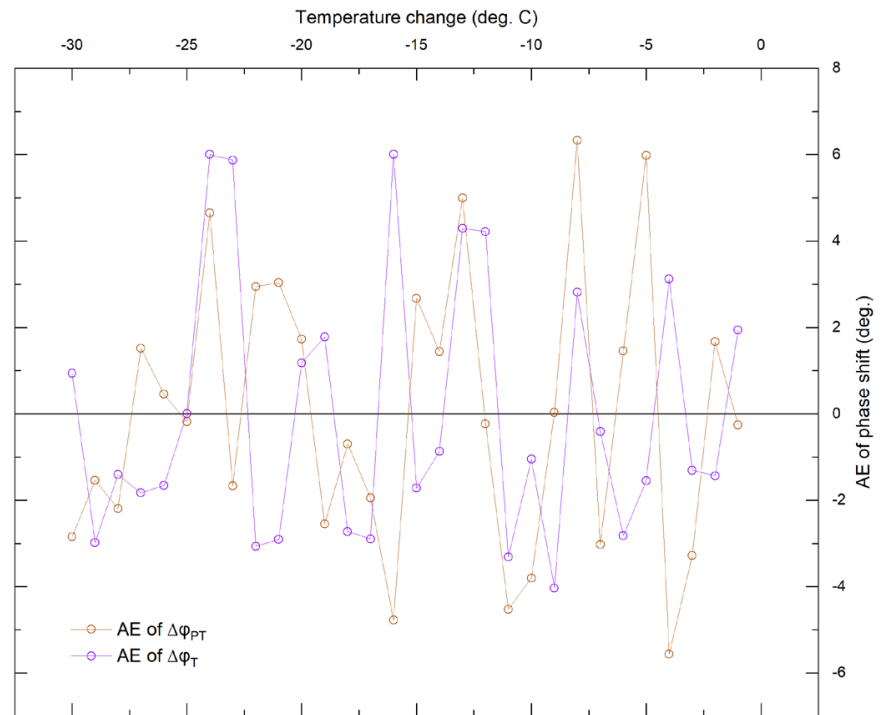

Fig. 11. Absolute errors (AE) of output phase shifts $\left(\Delta \varphi_{T}, \Delta \varphi_{P T}\right)$ depending on the temperature change $\Delta T$ at constant pressure $1 \mathrm{~atm}$ (Reference temperature: $\left.55^{\circ} \mathrm{C}\right)$.

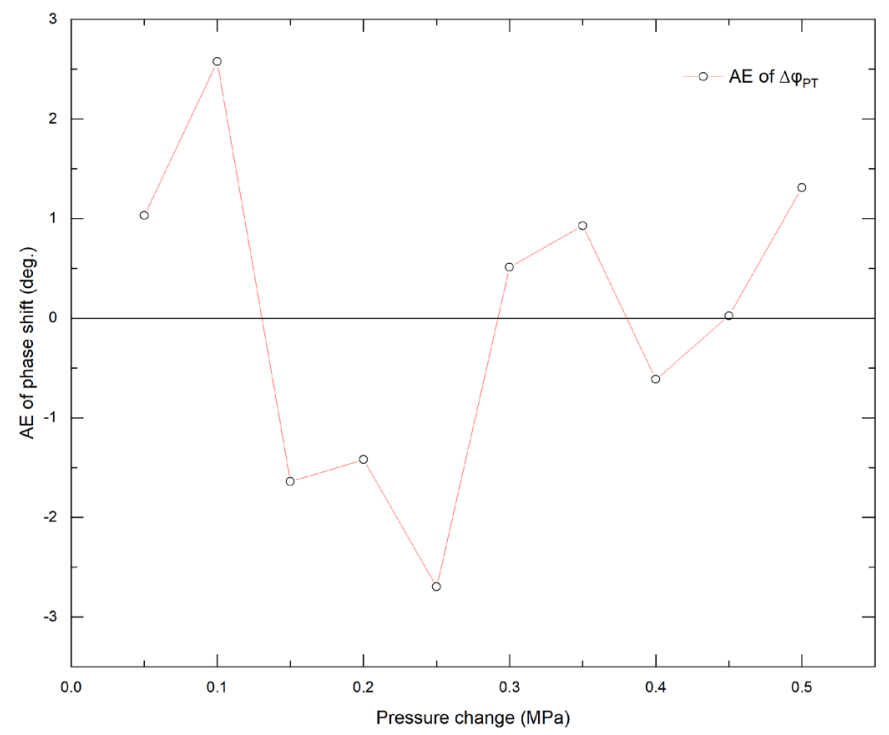

Fig. 12. Absolute errors (AE) of output phase shift $\Delta \varphi_{P T}$ depending on the pressure change $\Delta P$ at constant temperature $25{ }^{\circ} \mathrm{C}$ (Reference pressure: 1 atm).

Network Analyzer). The test result shows that the center frequency of the fabricated sensor node is $425.1220 \mathrm{MHz}$. In order to guarantee the quality of both interrogation and response signals to effectively extract sensing information, $1 \mu$ s width of the interrogation signal is selected. This is because the time delay from the IDT to every RDL is a multiple of approximately $1 \mu \mathrm{s}$. Less than $1 \mu \mathrm{s}$ width influences the sampling of the signals, while greater than $1 \mu$ s causes overlaps of response signals. Therefore, a $1 \mu$ s width $425.1220 \mathrm{MHz}$ RF pulse is selected as the interrogation signal. $20 \mu \mathrm{s}$ is selected as the period of the interrogation signal to avoid signal overlaps in two adjacent interrogation periods.

Fig. 7 shows the recorded waveform of the interrogation signal and three response signals reflected by three RDLs in time domain. Every response signal is independent and does not interfere with each other. The first RF pulse showing the largest

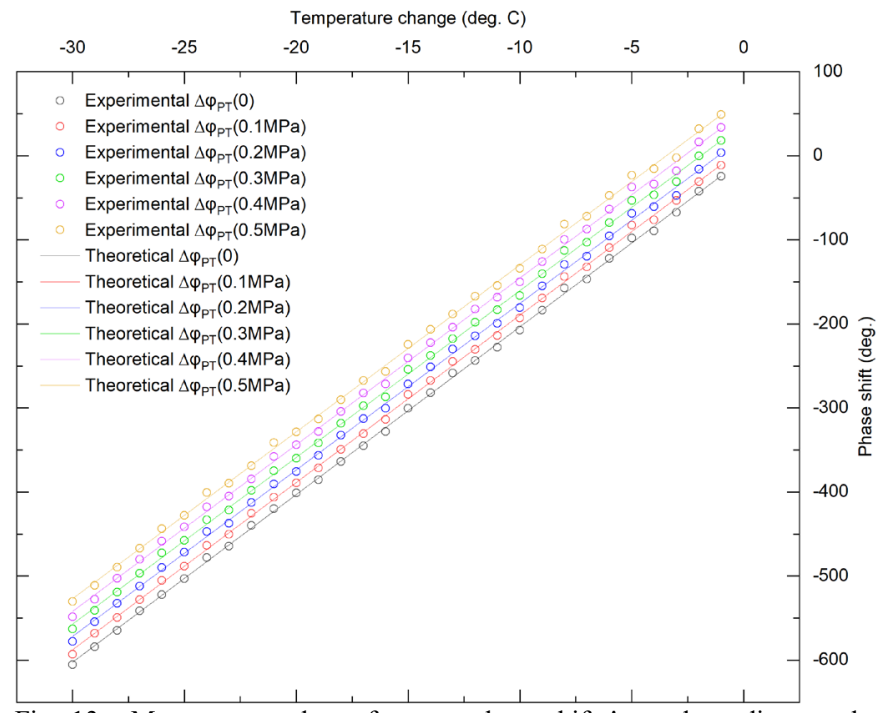

Fig. 13. Measurement data of output phase shift $\Delta \varphi_{P T}$ depending on the temperature change $\Delta T$ at different $\Delta P(0,0.1 \mathrm{MPa}, 0.2 \mathrm{MPa}, 0.3 \mathrm{MPa}, 0.4$ $\mathrm{MPa}, 0.5 \mathrm{MPa}$ ) (Reference temperature: $\left.55^{\circ} \mathrm{C}\right)($ Reference pressure: $1 \mathrm{~atm})$ (before temperature compensation).

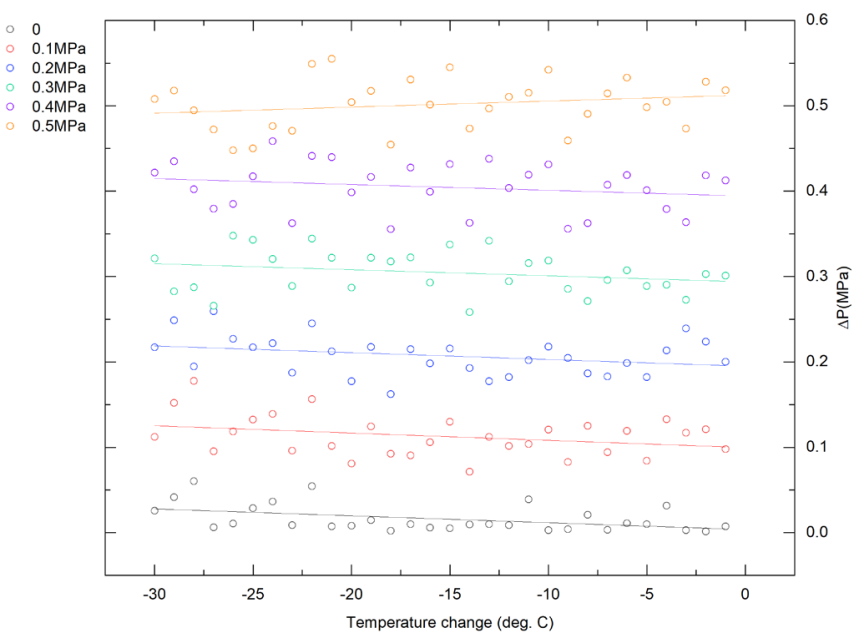

Fig. 14. Measurement value of $\Delta P$ depending on the temperature change $\Delta T$ at different actual $\Delta P(0,0.1 \mathrm{MPa}, 0.2 \mathrm{MPa}, 0.3 \mathrm{MPa}, 0.4 \mathrm{MPa}, 0.5 \mathrm{MPa})$ (Reference temperature: $55^{\circ} \mathrm{C}$ )(Reference pressure: $1 \mathrm{~atm}$ ) (After temperature compensation).

amplitude is the interrogation signal because the RF interrogation owns the largest power compared to three reflected RF response signals. The second RF pulse is the response signal reflected by $R_{2}$ because $R_{2}$ is the nearest reflector to the IDT. The third RF pulse is the response signal reflected by $R_{3}$ because $R_{3}$ is the second nearest reflector to the IDT, and the fourth RF pulse is the response signal reflected by $R_{1}$ because $R_{1}$ is the farthest reflector to the IDT compared to $\mathrm{R}_{2}$ and $\mathrm{R}_{3}$. The remaining low-amplitude RF pulses are highorder reflections between the IDT and three RDLs, and substrate edge reflections. These low-amplitude RF pulses are considered as interfering signals, and do not have any influences on the effective extraction of sensing information because of no overlaps in time domain with the first four effective RF pulses (interrogation signal and three response signals primarily reflected by the three RDLs).

The influence of interrogation distance (the distance between the reader and sensor node) on amplitudes of RF response 
TABLE II

RELATIVE ERRORS (RE) OF OUTPUT PHASE SHIFTS $\left(\Delta \varphi_{T}, \Delta \varphi_{P T}\right)$ DEPENDING ON THE TEMPERATURE CHANGE $\Delta T$ AT CONSTANT PRESSURE 1 ATM (REFERENCE

\begin{tabular}{|c|c|c|c|c|c|c|c|c|c|c|}
\hline$\Delta T\left({ }^{\circ} \mathrm{C}\right)$ & -1 & -2 & -3 & -4 & -5 & -6 & -7 & -8 & -9 & -10 \\
\hline $\operatorname{RE} \Delta \varphi_{P T}$ & $1.04 \%$ & $-3.94 \%$ & $4.88 \%$ & $6.21 \%$ & $-6.12 \%$ & $-1.19 \%$ & $2.06 \%$ & $-4.02 \%$ & $-0.02 \%$ & $1.84 \%$ \\
\hline $\operatorname{RE} \Delta \varphi_{T}$ & $-15.41 \%$ & $4.82 \%$ & $3.01 \%$ & $-5.90 \%$ & $2.17 \%$ & $3.27 \%$ & $0.42 \%$ & $-2.59 \%$ & $3.12 \%$ & $0.75 \%$ \\
\hline$\Delta T\left({ }^{\circ} \mathrm{C}\right)$ & -11 & -12 & -13 & -14 & -15 & -16 & -17 & -18 & -19 & -20 \\
\hline $\operatorname{RE} \Delta \varphi_{P T}$ & $1.98 \%$ & $0.09 \%$ & $-1.93 \%$ & $-0.51 \%$ & $-0.89 \%$ & $1.46 \%$ & $0.56 \%$ & $0.19 \%$ & $0.66 \%$ & $-0.43 \%$ \\
\hline $\mathrm{RE} \Delta \varphi_{T}$ & $2.13 \%$ & $-2.60 \%$ & $-2.44 \%$ & $0.45 \%$ & $0.82 \%$ & $-2.78 \%$ & $1.21 \%$ & $1.08 \%$ & $-0.68 \%$ & $-0.43 \%$ \\
\hline$\Delta T\left({ }^{\circ} \mathrm{C}\right)$ & -21 & -22 & -23 & -24 & -25 & -26 & -27 & -28 & -29 & -30 \\
\hline $\mathrm{RE} \Delta \varphi_{P T}$ & $-0.72 \%$ & $-0.67 \%$ & $0.36 \%$ & $-0.97 \%$ & $0.04 \%$ & $-0.09 \%$ & $-0.28 \%$ & $0.39 \%$ & $0.26 \%$ & $0.47 \%$ \\
\hline $\operatorname{RE} \Delta \varphi_{T}$ & $0.99 \%$ & $1.00 \%$ & $-1.88 \%$ & $-1.84 \%$ & $0.00 \%$ & $0.46 \%$ & $0.49 \%$ & $0.36 \%$ & $0.74 \%$ & $-0.23 \%$ \\
\hline
\end{tabular}

TABLE III

RELATIVE ERRORS (RE) OF OUTPUT PHASE SHIFT $\Delta \varphi_{P T}$ DEPENDING ON THE PRESSURE CHANGE $\Delta P$ AT CONSTANT TEMPERATURE $25^{\circ} \mathrm{C}$ (REFERENCE PRESSURE: 1

\begin{tabular}{|c|c|c|c|c|c|c|c|c|c|c|}
\hline$\Delta P(\mathrm{MPa})$ & 0.05 & 0.1 & 0.15 & 0.2 & 0.25 & 0.3 & 0.35 & 0.4 & $\overline{0.45}$ & $\overline{0.5}$ \\
\hline $\operatorname{RE} \Delta \varphi_{P T}$ & $14.84 \%$ & $16.44 \%$ & $-8.80 \%$ & $-5.45 \%$ & $-8.44 \%$ & $1.21 \%$ & $1.85 \%$ & $-1.10 \%$ & $0.04 \%$ & $1.82 \%$ \\
\hline
\end{tabular}

TABLE IV

ABSOLUTE ERRORS (AE) AND RELATIVE ERRORS (RE) OF MEASUREMENT VALUE OF $\Delta P(\mathrm{M} \Delta P)$ AT DIFFERENT ACTUAL $\Delta P($ A $\Delta P)($ REFERENCE PRESSURE: 1 ATM).

\begin{tabular}{cccccc}
$\mathrm{A} \Delta P(\mathrm{MPa})$ & 0 & 0.1 & 0.2 & 0.3 & 0.4 \\
$\mathrm{AE} \mathrm{M} \Delta P(\mathrm{MPa})$ & $1.62 \times 10^{-2}$ & $1.29 \times 10^{-2}$ & $7.29 \times 10^{-3}$ & $4.81 \times 10^{-3}$ & $4.78 \times 10^{-3}$ \\
$\mathrm{RE} \mathrm{M} \Delta P$ & 1 & $11.45 \%$ & $3.52 \%$ & $1.58 \%$ & $1.60 \times 10^{-3}$ \\
\hline
\end{tabular}

signals are shown in Fig. 8. Within $0.1 \mathrm{~m}$ interrogation distance, the response RF signal amplitudes are at high level. This range of interrogation distance is in favor of recording phase shifts of response RF signals. Therefore, the measurement data of phase shifts are recorded at $0.1 \mathrm{~m}$ interrogation distance. Beyond 0.1 $\mathrm{m}$ interrogation distance, the response RF signal amplitudes decrease dramatically. With the increase of interrogation distance, the response RF signal amplitudes fluctuate and decrease to 0 eventually. Within approximately $2.35 \mathrm{~m}$ interrogation distance, the WP-SAW sensor node is interrogatable, which means phase shifts can still be recorded but the interference of noise is strong. Beyond approximately $2.35 \mathrm{~m}$ interrogation distance, the response $\mathrm{RF}$ signal amplitudes sink into the noise and phase shifts cannot be recorded.

Fig. 9 shows the measurement data of temperature changes and phase shifts $\left(\Delta \varphi_{T}, \Delta \varphi_{P T}\right)$ at constant pressure 1 atm (Reference temperature: $55{ }^{\circ} \mathrm{C}$ ). Fig. 10 shows the measurement data of pressure changes and phase shift $\Delta \varphi_{P T}$ at constant temperature $25^{\circ} \mathrm{C}$ (Reference pressure: $1 \mathrm{~atm}$ ). Fig. 11 shows the absolute errors of temperature changes and phase shifts $\left(\Delta \varphi_{T}, \Delta \varphi_{P T}\right)$ at constant pressure 1 atm (Reference temperature: $55^{\circ} \mathrm{C}$ ). Fig. 12 shows the absolute errors of pressure changes and phase shift $\Delta \varphi_{P T}$ at constant temperature $25{ }^{\circ} \mathrm{C}$ (Reference pressure: $1 \mathrm{~atm}$ ). Table II shows the relative errors of temperature changes and phase shifts $\left(\Delta \varphi_{T}, \Delta \varphi_{P T}\right)$ at constant pressure 1 atm (Reference temperature: $55^{\circ} \mathrm{C}$ ). Table III shows the relative errors of pressure changes and phase shift $\Delta \varphi_{P T}$ at constant temperature $25{ }^{\circ} \mathrm{C}$ (Reference pressure: 1 atm). Absolute error is defined as equation (21), and relative error is defined as equation (22). Fig. 13 shows the measurement data of output phase shift $\Delta \varphi_{P T}$ and the temperature change $\Delta T$ at different $\Delta P(0,0.1 \mathrm{MPa}, 0.2 \mathrm{MPa}$, $0.3 \mathrm{MPa}, 0.4 \mathrm{MPa}, 0.5 \mathrm{MPa}$ ) (Reference temperature: 55 $\left.{ }^{\circ} \mathrm{C}\right)$ (Reference pressure: $1 \mathrm{~atm}$ ). Table IV shows the absolute and relative errors of measurement value of $\Delta P$ at different actual $\Delta P$ (Reference pressure: $1 \mathrm{~atm})$.

Absolute Error $(\mathrm{AE})=$ Experimental - Theoretical $(21)$

Relative Error $(\mathrm{RE})=\frac{\text { Absolute Error }(A E)}{\text { Experimental }}$

From Fig. 9 and Fig. 10, linearities of measurement data are shown. Therefore, fitting techniques can be utilized to analyze the theoretical functional relationship. Fig. 11 shows the range of absolute errors of temperature measurement is within approximately $\pm 7^{\circ}$. Fig. 12 shows the range of absolute errors of pressure measurement is within approximately $\pm 3^{\circ}$. Fig. 13 (measurement data of output phase shift $\Delta \varphi_{P T}$ ) reflects the features before temperature compensation, while Fig. 14 (measurement value of $\Delta P$ ) reflects the features after temperature compensation.

Utilizing fitting techniques, the variances of $\Delta \varphi_{T}$ and $\Delta \varphi_{P T}$ in Fig. 9 are 0.9997 and 0.9994 , and the variance of $\Delta \varphi_{P T}$ in Fig. 10 is 0.9946. All the variances of experimental data are close to 1, which means the experimental data of both Fig. 9 and 10 show good linearities and they fit the established functional relationship in Section IV. The functional relationship between the output phase shifts and the pressure change can be expressed as equation (23).

$\Delta P=\frac{1}{143.65} \Delta \varphi_{P T}-\frac{23}{143.65 * 12} \Delta \varphi_{T}+\frac{1.2681}{143.65}$

where $1 / 143.65$ and $23 /(143.65 * 12)$ are the constants $M$ and $N$ in equation (20), and 1.2681/143.65 is the correction.

The sensor node has a good performance in the range of 
pressure difference from 0 to $0.5 \mathrm{MPa}$, which meets the normal 28 -meter water pressure sensing requirements. The accuracy of this sensor is $7.22 \mathrm{kPa}$.

\section{CONCLUSIONS}

This paper established the functional relationship between output phase shifts and the pressure change for the temperaturecompensated bidirectional WP-SAW RDL pressure sensor node. It was tested by an experimental framework which simulates a water pressure sensing adaptor. The experimental results: the experimental data show good linearities, which fits the established functional relationship; the numerical functional relationship has been derived and expressed; the sensor node has a good performance in the range of pressure difference from 0 to $0.5 \mathrm{MPa}$, which meets the normal 28 -meter water pressure sensing requirements; the accuracy of this sensor is $7.22 \mathrm{kPa}$. Overall, this WP-SAW sensor node can be utilized for the water pressure sensing tasks in water distribution systems.

\section{REFERENCES}

[1] R. Liemberger and A. Wyatt, "Quantifying the global non-revenue water problem," Water Science and Technology: Water Supply, vol. 19(3), pp. 831-837, 2018.

[2] A. O. Lambert, "International report: water losses management and techniques," Water Science and Technology: Water Supply, vol. 2, pp. 120, 2002.

[3] R. Mamlook and O. Al-Jayyousi, "Fuzzy sets analysis for leak detection in infrastructure systems: a proposed methodology," Clean Technologies and Environmental Policy, vol. 6, pp. 26-31, 2003.

[4] J. A. Goulet, S. Coutu and I. F. C. Smith, "Model falsification diagnosis and sensor placement for leak detection in pressurized pipe networks," Journal of Advanced Engineering Informatics, vol. 27, pp. 261-269, 2013.

[5] A. F. Colombo, "Energy and costs of leaky pipes: toward comprehensive picture," Journal of Water Resources Planning and Management, vol. 128, pp. 441-450, 2002.

[6] R. Puust, Z. Kapelan, D.A. Savic and T. Koppel, "A review of methods for leakage management in pipe networks," Urban Water Journal, vol. 7, pp. $25-45,2010$.

[7] J. Wang, Z. Tang, Y. Gui, W. Wu, and M. Zhao, "Model and application of pressure-dependent leakage detection in water distribution network," The $4^{\text {th }}$ International Conference on Civil, Architectural and Hydraulic Engineering (ICCAHE2015), Guangzhou, China, June 20-21, 2015, Progress in Civil, Architectural and Hydraulic Engineering IV: Hydraulic Engineering, pp. 795-801, 2016.

[8] J. Harmouche and S. Narasimhan, "Long-term monitoring for leaks in water distribution networks using association rules mining," IEEE Transactions on Industrial Informatics, vol. 16(1), pp. 258-266, 2020.

[9] J. Kang, Y. Park, J. Lee, S. Wang, and D. Eom, "Novel leakage detection by ensemble CNN-SVM and graph-based localization in water distribution systems," IEEE Transactions on Industrial Electronics, vol. 65(5), pp. 4279-4289, 2018.

[10] A. Pohl, "A review of wireless SAW sensors," IEEE Transactions on Ultrasonics, Ferroelectrics, and Frequency Control, vol. 47, pp. 317-332, 2000.

[11] D. C. Malocha, M. Gallagher, B. Fisher, J. Humphries, D. Gallagher and N. Kozlovski, "A passive wireless multi-sensor SAW technology device and system perspectives," Sensors, vol. 13, pp. 5897-5922, 2013.

[12] W. E. Bulst, G. Fischerauer and L. Reindl, "State of the art in wireless sensing with surface acoustic waves," IEEE Transactions on Industrial Electronics, vol. 48(2), pp. 265-271, April 2001.

[13] B. Drafts, "Acoustic wave technology sensors," IEEE Transactions on Microwave Theory and Techniques, vol. 49(4), pp. 795-802, 2001.

[14] L. Xie, T. Wang, J. Xing, and X. Zhu, "An embedded surface acoustic wave pressure sensor for monitoring civil engineering structures," IEEE Sensors Journal, vol. 18(13), pp. 5232-5237, 2018.
[15] J. Devkota, D. W. Greve, T. Hong, K.-J. Kim, and P. R. Ohodnicki, “An $860 \mathrm{MHz}$ wireless surface acoustic wave sensor with a metal-organic framework sensing layer for $\mathrm{CO}_{2}$ and $\mathrm{CH}_{4}$," IEEE Sensors Journal, vol. 20(17), pp. 9740-9747, 2020.

[16] B. V. Sveshnikov, and A. P. Shitvov, "Multitransducer SAW device architecture for passive wireless sensor tags," IEEE Transactions on Ultrasonics, Ferroelectrics, and Frequency Control, vol. 65(11), pp. 2176-2183, 2018.

[17] R. Shi, J. Ruan, J. Lv, C. Zhang, T. Han, P. Qin, P. Li, and Y. Wen, "Dualfrequency interrogation and hierarchical evaluation scheme for SAW reflective delay-line sensors," IEEE Transactions on Ultrasonics, Ferroelectrics, and Frequency Control, vol. 67(6), pp. 1258-1266, 2020.

[18] V. Kalinin, A. Stopps, J.- F. Chacon, E. Artigao, V. Kappatos, C. Selcuk, T.-H. Gan, D. Lekou and R. Ginige, "Novel torque sensor design and enhanced envelope method of acoustic emission signals in condition monitoring of the nacelle in wind turbine," Proceedings of the $11^{\text {th }}$ International Conference on Condition Monitoring and Machinery Failure Prevention Technologies, Manchester, UK, 10-12 June 2014.

[19] V. Kalinin, A. Leigh, A. Stopps and S. B. Hanssen, "SAW torque sensor for marine applications," Proceedings of 2017 Joint Confonerence of the IEEE International Frequency Control Symposium and European Frequency and Time Forum, Besancon, France, pp. 347-352, 9-13 July, 2017.

[20] V. Kalinin, "Wireless physical SAW sensors for automotive applications," Proceedings of 2011 IEEE International Ultrasonic Symposium, Orlando, USA, pp. 212-221, October 18-22, 2011.

[21] V. Kalinin, "Wireless passive resonant SAW sensors for monitoring temperature, strain, torque, and pressure," Proceedings of VII ECCOMAS Thematic Conference on Smart Structures and Materials SMART 2017, Madrid, Spain, pp. 1453-1464, 6-8 June 2017.

[22] Y. Yang, C. Zhang, Q. Shan, P. Qin, T. Han, Y. Wen, and P. Li, “Adaptive environment-aware threshold for passive and wireless SAW resonant sensors," IEEE Sensors Journal, vol. 19(20), pp. 9394-9401, 2019.

[23] M. Khoshakhlagh and N. Masoumi, "A pigeonhole principle-based method for estimating the resonant frequency of SAWR sensors," IEEE Transactions on Instrumentation and Measurement, vol. 68(11), pp. 4502-4509, 2019.

[24] F. Lurz, S. Lindner, S. Linz, S. Mann, R. Weigel, and A. Koelpin, "Highspeed resonant surface acoustic wave instrumentation based on instantaneous frequency measurement," IEEE Transactions on Instrumentation and Measurement, vol. 66(5), pp. 974-984, 2017.

[25] Z. Tang, W. Wu, J. Gao, and P. Yang, "SAW delay line based IoT smart sensing in water distribution system," 2018 IEEE 20th International Conference on High Performance Computing and Communications; IEEE 16th International Conference on Smart City; IEEE 4th International Conference on Data Science and Systems (HPCC/SmartCity/DSS), Exeter, UK, pp. 1474-1478, 28-30 June 2018.

[26] Z. Tang, W. Wu, J. Gao, and P. Yang, "Feasibility study on wireless passive SAW sensor in IoT enabled water distribution system," 2017 IEEE International Conference on Internet of Things (iThings) and IEEE Green Computing and Communications (GreenCom) and IEEE Cyber, Physical and Social Computing (CPSCom) and IEEE Smart Data (SmartData), Exeter, UK, pp. 830-834, 21-23 June 2017.

[27] Z. Tang, W. Wu, J. Gao, P. Yang, J. Luo, and C. Fu, "Water pressure monitoring using a temperature-compensated WP-SAW pressure sensor," The $18^{\text {th }}$ IEEE International Conference on Industrial Informatics, University of Warwick, Coventry, UK, vol. 1, pp. 354-357, 20-23 July, 2020.

[28] Z. Tang, W. Wu, J. Gao, J. Luo, R. Tao, C. Fu, and L. Xu, "A multiiteration enhanced 2P-SMA method for improved error reduction on a WP-SAW water temperature and pressure sensor," IEEE Access, vol. 9, pp. 48236-48243, 2021.

[29] Z. Tang, W. Wu, and J. Gao "Water pressure sensing based on wireless passive SAW technology," The 13th International Conference on Computing and Control for the Water Industry (CCWI2015), De Montfort University, Leicester, UK, 2-4 September 2015, Procedia Engineering, vol. 119, pp. 892-900, 2015. 